\title{
Venous thromboembolism risk assessment and prophylaxis in hospitalised medical patients in the Cape Town metropole, South Africa
}

\author{
A Wehmeyer, BPharm (D); R Coetzee, MPharm, PharmD; J McCartney, MScPharm, PhD \\ School of Pharmacy, Faculty of Natural Sciences, University of the Western Cape, Cape Town, South Africa
}

Corresponding author: A Wehmeyer(3470695@myuwc.ac.za)

Background. Venous thromboembolism (VTE) is regarded as the most preventable cause of inpatient death in hospital settings globally. VTE can be prevented through the provision of non-pharmacological and/or pharmacological thromboprophylaxis following individualised risk screening. The Caprini risk assessment model (RAM) offers a validated and well-established approach for VTE risk assessment in medical inpatients. Literature findings describe a trend towards inappropriate and under-prescribing of thromboprophylaxis in this population. Together with concerns regarding clinicians' perceived importance of VTE risk assessment, the need to clarify these aspects of practice is evident.

Objectives. To describe VTE risk assessment and prophylaxis practices of medical practitioners in public sector hospitals in Western Cape Province, South Africa (SA).

Methods. A retrospective, cross-sectional study design was employed in the medical wards of two district hospitals and one regional hospital in the Cape Town metropole, Western Cape. Medical folders of adult medical inpatients admitted between January and July 2020 were reviewed to assess VTE risk using the Caprini RAM. Thromboprophylaxis therapy prescribed and contraindications to chemoprophylaxis were also evaluated.

Results. Of 380 patients included in the review, 51.6\% were female, and the average age was 52.1 years (range 18 - 96); 21.3\% had their weight recorded, while none had their height documented. Infectious disease was the predominant diagnosis $(49.2 \%)$ detected in the sample. Common VTE risk factors identified included bed rest/restricted mobility for $<72$ hours $(76.3 \%)$ and serious infection $(67.4 \%)$. A total of $97.1 \%$ of patients $(n=369)$ were found to be at moderate or higher risk of VTE (Caprini score $\geq 2$ ). Of this at-risk group, $24.1 \%$ were eligible to receive chemoprophylaxis, yet no prescription for thromboprophylaxis was identified. Seventy percent of patients $(n=266)$ were prescribed chemoprophylaxis, with enoxaparin accounting for $98.5 \%$ of regimens. Contraindications to chemoprophylaxis were recorded in $13.4 \%$ of patients.

Conclusions. Although rates of VTE prophylaxis in medical inpatients may be improving, thromboprophylaxis still remains critically underutilised in this population. This study highlighted a consequence of this trend, with inappropriate chemoprophylaxis prescribing becoming more evident. Mechanical prophylaxis prescribing in medical inpatients is lacking, despite the associated benefits. RAMs should be adapted for the SA setting, where infectious diseases are prevalent. Future research should assess RAM use by clinicians, as this could provide insight into improving RAM uptake and thromboprophylaxis prescribing.

S Afr Med J 2022;112(2):117-123. https://doi.org/10.7196/SAMJ.2022.v112i2.16040

Venous thromboembolism (VTE) is a preventable and potentially life-threatening disease that frequently complicates the admission of hospitalised patients. VTE can manifest as deep-vein thrombosis (DVT) and/or pulmonary embolism (PE), which are both associated with increased morbidity and mortality. PE as a complication of VTE is the most preventable cause of inpatient death across the globe. ${ }^{[1,2]}$ VTE is linked to increased healthcare costs, intensive care unit admission and longer hospital stay. ${ }^{[3]}$

Findings from the multinational ENDORSE (Epidemiologic International Day for the Evaluation of Patients at Risk for Venous Thromboembolism in the Acute Hospital Care Setting) study revealed that more than half of all hospitalised patients were at risk for VTE, and $41.5 \%$ of these were medical inpatients. ${ }^{[4]} \mathrm{A}$ multinational, crosssectional survey conducted across five countries in sub-Saharan Africa found that a greater proportion of medical inpatients were at risk for VTE (62.3\%) compared with surgical patients (43.8\%). ${ }^{[5]}$

In South Africa (SA), studies aimed at assessing VTE risk are limited, so there is a paucity of VTE-related data in the SA population. ${ }^{[6,7]}$ This lack of data extends across both the private and public healthcare sectors. ${ }^{[2,3]}$ The SA-based TUNE-IN (The Use of VTE prophylaxis in relatioN to patiEnt risk profiling) study aimed to assess VTE prophylaxis use in hospitalised patients in relation to their risk profile. The authors reported a $67.1 \%$ risk of VTE in medical inpatients admitted to private healthcare sector hospitals across Gauteng Province, SA. ${ }^{[8]}$

VTE can be prevented through the provision of appropriate non-pharmacological and/or pharmacological prophylaxis following individualised patient screening with a structured risk assessment model (RAM) or through clinical evaluation. ${ }^{[3,9]}$ The Caprini RAM offers a simple and comprehensive approach to VTE risk assessment in both surgical and medical inpatients. ${ }^{[1-3]}$ Furthermore, it has undergone several modifications since its introduction in 1991 and has been validated in $>250000$ patients in $>100$ trials worldwide. ${ }^{[1]}$ Development of the Caprini RAM was based on the implementation of the American College of Chest Physicians (ACCP) thromboprophylaxis guideline, one of the leading VTE prophylaxis 
guidelines worldwide. ${ }^{[3]}$ The therapeutic practice guideline for VTE developed by the Southern African Society of Thrombosis and Haemostasis closely mirrors the ACCP guideline, which bases VTE risk assessment on the Caprini RAM. ${ }^{[2,9]}$

SA has one of the greatest dual burdens of tuberculosis (TB) and HIV infection globally, and both infections have well-established relationships with VTE development. ${ }^{[7]}$ In SA, TB was reported to be the leading cause of death in 2017, and disruptions in TB-related care due to the COVID-19 pandemic have been reported. ${ }^{[10]}$ SA has the largest HIV epidemic globally, with $19 \%$ of all persons infected with the virus living in that country. ${ }^{[11]} \mathrm{TB}$ and HIV are prominent VTE risk factors that are frequently overlooked in the SA setting, and their impact is not well known. ${ }^{[12]} \mathrm{A}$ prospective cohort study conducted at Charlotte Maxeke Johannesburg Academic Hospital aimed to investigate the association of VTE with TB and HIV. The authors found that $53.0 \%$ and $21.2 \%$ of patients presenting with DVT were infected with HIV and TB, respectively. ${ }^{[7]}$

Low-molecular-weight heparin (LMWH) and unfractionated heparin have proved to be safe, effective and cost-effective agents for VTE prophylaxis in medical inpatients. ${ }^{[12]}$ Mechanical thromboprophylaxis, including intermittent pneumatic compression (IPC) and graduated compression stockings (GCSs), are advocated for use in patients who have contraindications to anticoagulants. ${ }^{[9,13]}$

Despite growing evidence supporting VTE risk assessment and thromboprophylaxis in medical inpatients, inappropriate and underprescribing of thromboprophylaxis is evident. ${ }^{[4,14]}$ The multinational survey conducted by Kingue et al. ${ }^{[5]}$ found that only $36.2 \%$ of medical inpatients who were at risk for VTE received prophylaxis. ${ }^{[5]}$ In SA, a study investigating thromboprophylaxis in a private hospital group, which included 373020 patients, found that $<25 \%$ of at-risk patients received guideline-appropriate interventions. ${ }^{[3]}$

In SA's public healthcare sector, a lack of adequate data regarding VTE risk assessment and prophylaxis practices in medical inpatients has been reported. ${ }^{[6]}$ Together with the low rate of adherence to VTE clinical practice guidelines, this accentuates the need to clarify these aspects of practice. ${ }^{[4]}$

\section{Objectives}

To describe the VTE risk assessment and prophylaxis practices of medical practitioners in public sector hospitals in the Cape Town metropole, Western Cape Province, South Africa.

\section{Methods}

A quantitative, observational, descriptive, exploratory crosssectional design was employed in this study. Quantitative data were retrospectively retrieved from patient medical folders in the medical wards of three public sector hospitals (two district hospitals and one regional hospital) in the Cape Town metropole.

Only folders of adult ( $\geq 18$ years) medical inpatients who were admitted to medical wards between January and July 2020 were included in the study. Patients aged $<18$ years, surgical patients, and those who required therapeutic anticoagulation for confirmed or suspected VTE, atrial fibrillation, acute coronary syndromes, or any other reason besides VTE prophylaxis were excluded from the study.

The estimated proportion of medical inpatients with VTE in SA was reported to be $57.1 \%{ }^{[8]}$ Using this proportion and a two-sided confidence interval of $95 \%$ with a $\pm 5 \%$ margin of error, a minimum of 377 medical folders needed to be reviewed to achieve an adequate sample size. Length of hospital stay for each patient was calculated in units of 24 hours and reported in days. ${ }^{[15]}$

Convenience sampling was used to recruit hospitals for inclusion in the study, where the first hospitals to respond to the recruitment notice were selected. Patient medical folders were selected using random systematic selection. This was achieved through the randomisation of a list of medical folder numbers obtained from each facility's electronic continuity of care record (eCCR). Patient medical folder numbers were then selected from this list at fixed, periodic intervals, prior to being accessed at each participating hospital.

Data were manually extracted from medical folders with the use of a predesigned data collection tool, which included an updated version of the Caprini RAM. ${ }^{[1]}$ Prior to data collection, the tool was piloted to further inform and refine its design. Patients' individual VTE risk factors were documented, followed by the calculation of their VTE risk score and subsequent VTE risk categorisation according to the Caprini RAM. Inpatient prescription charts were also evaluated to compare thromboprophylaxis prescribed with that recommended by the Caprini RAM. Additional data collected included basic demographic information. Data were then exported to a structured Microsoft Office Excel 2016 spreadsheet (Microsoft, USA), from which analyses were performed.

Descriptive statistics were used to summarise data in the form of percentages and proportions. Student's $t$-test was used to draw comparisons between means for gender differences in terms of age, weight, height and Caprini VTE risk scores, and $p$-values $<0.05$ were regarded as significant.

\section{Ethical considerations}

Approval to conduct the study was granted by the University of the Western Cape's Biomedical Research Ethics Committee (ref. no. BM20/5/9) and Western Cape Government Health (ref. no. WC_202007_013). Informed consent from patients was not required by the applicable ethics committees because the study was retrospective and lacked direct patient contact, and data were anonymised prior to analysis.

\section{Results}

The review included 435 medical folders, which were randomly selected from 4884 medical admissions that were registered on the eCCR database during the 7-month data collection period. Of these, 380 were included in the final sample of the study. Forty-one medical folders were excluded because they did not meet inclusion criteria and 14 folders were excluded owing to missing information.

The patient sample consisted of more females (51.6\%) than males $(48.4 \%)$, with an overall mean age of 52.1 years (Table 1). No significant difference between the mean age of males and females was detected in the sample $(p=0.25)$.

Only 81 patients $(21.3 \%)$ had a documented weight, but not one record of patient height was documented, so body mass index (BMI) could not be calculated. Among these 81 patients, no statistically significant difference between the mean documented weights was detected between males and females $(p=0.94)$. The average length of stay was calculated at 5.9 days (range $1-35$ ), with $>80 \%$ of patients hospitalised for $\geq 3$ days.

Approximately a quarter of the patients had been hospitalised within 3 months prior to admission. Evidence of recent hospitalisation ( $\leq 90$ days) is shown in Fig. 1 .

The most frequently documented diagnosis was infectious disease (49.2\%). This was followed by neurological disease, which accounted for $14.5 \%$ of the total sample. Various forms of TB as well as polymerase chain reaction (PCR)-positive and clinically diagnosed/ highly suspected COVID-19 infections accounted for $17.7 \%$ and $11.8 \%$ of the sample, respectively. Table 2 provides an overview of the diagnoses identified among sampled patients. 
Table 1. Demographics of the study population $(N=380)$ Patients,

Age (years)

\begin{tabular}{|c|c|c|c|c|c|c|c|c|c|c|}
\hline \multirow{2}{*}{ Gender } & \multirow{2}{*}{$n(\%)$} & \multirow{2}{*}{\multicolumn{2}{|c|}{$18-30, n(\%) \quad 31-40, n(\%)$}} & & \multirow[b]{2}{*}{ Mean (SD) } & \multirow[b]{2}{*}{ Minimum } & \multirow[b]{2}{*}{ Maximum } & \multirow[b]{2}{*}{ Median } \\
\hline & & & & $41-60, n(\%)$ & $61-74, n(\%)$ & $\geq 75, n(\%)$ & & & & \\
\hline Female & $196(51.6)$ & $39(10.3)$ & $34(8.9)$ & $54(14.2)$ & $38(10.0)$ & $31(8.2)$ & $53.1(18.0)$ & 18 & 96 & 55 \\
\hline Male & $184(48.4)$ & $19(5)$ & $42(11.1)$ & $69(18.2)$ & $43(11.3)$ & $11(2.9)$ & $51.0(15.3)$ & 19 & 84 & 52 \\
\hline Total & $380(100)$ & $58(15.3)$ & $76(20.0)$ & $123(32.4)$ & $81(21.3)$ & $42(11.1)$ & $52.1(16.8)$ & 18 & 96 & 54 \\
\hline
\end{tabular}

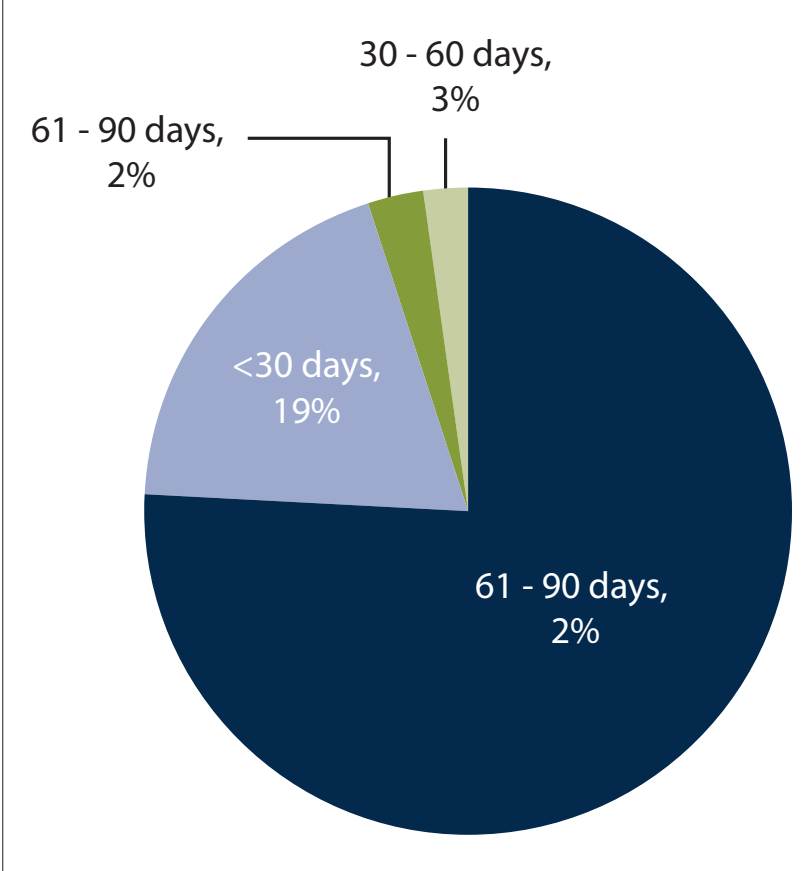

Fig. 1. Previous hospitalisation in the patient sample $(\mathrm{N}=380)$.

The most common VTE risk factors identified were: (i) patients currently at bed rest/restricted mobility for $<72$ hours (76.3\%); (ii) serious infection that required hospitalisation and antibiotics (67.4\%); (iii) age 41 - 60 years (32.9\%); and (iv) a personal or family history of genetic or acquired thrombophilia (27.6\%).

Other notable risk factors identified in the sample included being non-ambulatory for $>72$ hours (20.8\%) and age $61-74$ years $(20.3 \%)$. The distribution of VTE risk factors among sampled patients in relation to the Caprini RAM risk scores is summarised in Table 3.

Following application of the Caprini RAM, 97.1\% of patients ( $n=369)$ were identified to be at moderate or higher risk of VTE (Caprini score $\geq 2$ ). No significant difference in Caprini VTE risk scores was detected between males and females $(p=0.91)$. Thromboprophylaxis was prescribed for $71.0 \%(n=262)$ of the 369 patients in the at-risk group, for $75.4 \%(n=180)$ of the 239 patients in the highest-risk group (Caprini score $\geq 5$ ), and for $65.9 \%(n=60)$ of the 91 patients in the high-risk group (Caprini score 3 - 4). The number of patients in each Caprini risk score category and the rate of thromboprophylaxis prescription are shown in Table 4.

A total of 266 patients (70.0\%) were prescribed thromboprophylaxis, in all cases a subcutaneously administered anticoagulant. No prescription for any form of mechanical prophylaxis was documented. Table 5 summarises the initial chemoprophylactic agents prescribed. Enoxaparin was the most commonly prescribed thromboprophylactic agent ( $98.5 \%$ of cases; $n=262$ ).

Only 41 of the 266 patients who were prescribed thromboprophylaxis had documented changes to their initial regimen. Twelve percent $(n=32)$ of the sample who were prescribed thromboprophylaxis

\begin{tabular}{|c|c|}
\hline Primary diagnosis & $n(\%)$ \\
\hline Infectious disease & $187(49.2)$ \\
\hline $\begin{array}{l}\text { Clinically diagnosed/highly suspected COVID-19 } \\
\text { infection }\end{array}$ & $7(1.8)$ \\
\hline Community-acquired pneumonia & $22(5.8)$ \\
\hline Disseminated TB & $20(5.3)$ \\
\hline PCR-positive COVID-19 & $38(10.0)$ \\
\hline Pulmonary TB & $42(11.1)$ \\
\hline TB meningitis & $5(1.3)$ \\
\hline Unspecified lower respiratory tract infection & $5(1.3)$ \\
\hline Urinary tract infection & $20(5.3)$ \\
\hline Other infectious diseases & $28(7.4)$ \\
\hline Neurological disease & $55(14.5)$ \\
\hline Epilepsy & $9(2.4)$ \\
\hline Ischaemic stroke & $21(5.5)$ \\
\hline Unspecified stroke & $5(1.3)$ \\
\hline Other neurological diseases & $20(5.3)$ \\
\hline Gastrointestinal/hepatobiliary disease & $35(9.2)$ \\
\hline Acute gastroenteritis & $20(5.3)$ \\
\hline Other gastrointestinal/ hepatobiliary diseases & $15(4.0)$ \\
\hline Cardiovascular disease & $27(7.1)$ \\
\hline Acute decompensated heart failure & $22(5.8)$ \\
\hline Other cardiovascular diseases & $5(1.3)$ \\
\hline Pulmonary disease & $26(6.8)$ \\
\hline Acute exacerbation of COPD & $20(5.3)$ \\
\hline Other pulmonary diseases & $6(1.6)$ \\
\hline Endocrine/metabolic disease & $17(4.5)$ \\
\hline Diabetic ketoacidosis & $10(2.6)$ \\
\hline Other endocrine/metabolic diseases & $7(1.8)$ \\
\hline Malignancy (active)/haematological disease & $14(3.7)$ \\
\hline Bronchogenic carcinoma & $8(2.1)$ \\
\hline Other malignancies/haematological diseases & $6(1.6)$ \\
\hline Renal disease & $10(2.6)$ \\
\hline Acute kidney injury & $7(1.8)$ \\
\hline Other renal diseases & $3(0.8)$ \\
\hline Psychiatric diseases & $7(1.8)$ \\
\hline Drug overdose & $6(1.6)$ \\
\hline Other psychiatric diseases & $1(0.3)$ \\
\hline Other & $3(0.8)$ \\
\hline \multicolumn{2}{|c|}{$\begin{array}{l}\mathrm{TB}=\text { tuberculosis; } \mathrm{PCR}=\text { polymerase chain reaction; } \\
\mathrm{COPD}=\text { chronic obstructive pulmonary disease. } \\
\text { *Clinically diagnosed or high suspicion of COVID-19 based on chest radiograph and } \\
\text { clinical examination. }\end{array}$} \\
\hline
\end{tabular}

were switched to an alternative regimen, the majority $(n=8)$ being switched to a lower dose of enoxaparin (20 mg 24-hourly). Three percent $(n=9)$ of those with documented changes had their thromboprophylaxis regimen discontinued entirely. Table 6 displays the altered thromboprophylaxis regimens prescribed.

Contraindications to chemoprophylaxis were recorded in $13.4 \%$ of patients in the total sample $(n=51)$. Active bleeding as a contraindication included any documented active haemoptysis, 
Table 3. Distribution of VTE risk factors in the study population in accordance with Caprini $\operatorname{RAM}^{[1]}(N=380)^{\star}$

\begin{tabular}{|c|c|c|c|c|}
\hline 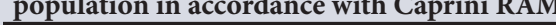 & & 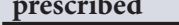 & & \\
\hline VTE risk factors score on Caprini RAM & $\begin{array}{l}\text { Risk factors, } \\
n \text { (\% of risk } \\
\text { factors) }\end{array}$ & $\begin{array}{l}\text { Caprini VTE } \\
\text { risk score }\end{array}$ & Patients, $n(\%)$ & $\begin{array}{l}\text { Patients prescribed } \\
\text { thromboprophylaxis, } \\
n(\%)\end{array}$ \\
\hline 1 point & & Low $(0-1)$ & $11(2.9)$ & $4(1.5)$ \\
\hline Age 41 - 60 years & $125(32.9)$ & Moderate (2) & $39(10.3)$ & $22(8.3)$ \\
\hline Minor surgery planned ( $<45$ minutes) & $2(0.5)$ & High $(3-4)$ & $91(23.9)$ & $60(22.6)$ \\
\hline Past major surgery ( $>45$ minutes) within past & $4(1.1)$ & Highest $(\geq 5)$ & $239(62.9)$ & $180(67.7)$ \\
\hline 30 days & & Total & $380(100)$ & $266(100)$ \\
\hline Visible varicose veins & $3(0.8)$ & $\mathrm{VTE}=$ venous thr & & \\
\hline History of inflammatory bowel disease & 0 & & & \\
\hline
\end{tabular}

$56(14.7)$

BMI $>25 \mathrm{~kg} / \mathrm{m}^{2 \dagger} \quad 47(12.4)$

Myocardial infarction $2(0.5)$

Congestive heart failure $\quad 39(10.3)$

Serious infection (requires hospitalisation and $256(67.4)$

antibiotic(s))

Chronic respiratory disease, e.g. COPD $60(15.8)$

Currently at bed rest or restricted mobility, $\quad 290(76.3)$

including the use of removable leg brace for

$<72$ hours

Current use of birth control therapy or hormone 0

replacement therapy

Pregnant or conceived within the past 30 days $3(0.8)$

History of unexplained stillborn infant, recurrent $1(0.3)$

spontaneous abortion $(\geq 3)$, premature birth with

toxaemia or growth-restricted infant

2 points

Age 61 - 74 years $78(20.5)$

Current or past malignancies (excluding skin $\quad 15(3.9)$

cancer but including melanoma)

Planned major surgery lasting longer than $\quad 5(1.3)$

45 minutes (including laparoscopic and

arthroscopic)

Non-removable plaster cast that prevents leg

movement within the past 30 days

Tube in blood vessel in neck or chest that delivers 0

blood or medicine directly to the heart within the

last month (e.g. central venous access)

Confined to bed for $\geq 72$ hours (unable to

$79(20.8)$

ambulate continuously for 30 feet $(9 \mathrm{~m})$

3 points

Age $\geq 75$ years

$44(11.6)$

History of thrombosis: DVT, PE or superficial $\quad 9(2.4)$

venous thrombosis

Family history of thrombosis (up to third-degree 0

relatives)

Personal or family history of genetic or acquired $105(27.6)$

thrombophilia

5 points

Elective major lower extremity arthroplasty $\quad 1(0.3)$

Hip, pelvis or leg fracture $1(0.3)$

Multiple trauma $\quad 1(0.3)$

Spinal cord injury with resultant paralysis $3(0.8)$

Stroke $29(7.6)$

VTE $=$ venous thromboembolism; $\mathrm{RAM}=$ risk assessment model;

$\mathrm{BMI}=$ body mass index; $\mathrm{COPD}=$ chronic obstructive pulmonary disease;

DVT $=$ deep-vein thrombosis; $\mathrm{PE}=$ pulmonary embolism.

${ }^{\star}$ Total risk factors identified in sample, $n=1258$.

${ }^{\top}$ BMI recorded as a risk factor when documented as being elevated in clinical notes.
Table 4. Risk stratification and thromboprophylaxis prescribed

\begin{tabular}{|c|c|c|c|}
\hline Agent & Dose & Frequency & $n(\%)$ \\
\hline \multirow[t]{11}{*}{ Enoxaparin SC } & $20 \mathrm{mg}$ & 24-hourly & $12(4.5)$ \\
\hline & & 48-hourly & $1(0.4)$ \\
\hline & $40 \mathrm{mg}$ & 24-hourly & $210(78.9)$ \\
\hline & & 12-hourly & $3(1.1)$ \\
\hline & & 48-hourly & $1(0.4)$ \\
\hline & $50 \mathrm{mg}$ & 24-hourly & $1(0.4)$ \\
\hline & $60 \mathrm{mg}$ & 24-hourly & $12(4.5)$ \\
\hline & & 12-hourly & $3(1.1)$ \\
\hline & $80 \mathrm{mg}$ & 24-hourly & $4(1.5)$ \\
\hline & & 12-hourly & $13(4.9)$ \\
\hline & $100 \mathrm{mg}$ & 12-hourly & $2(0.8)$ \\
\hline UFH SC & $5000 \mathrm{IU}$ & 8-hourly & $4(1.5)$ \\
\hline
\end{tabular}

Table 6. Alternative thromboprophylaxis regimens

\begin{tabular}{llll}
\hline Agent & Dose & Frequency & $\boldsymbol{n}(\%)$ \\
\hline Enoxaparin SC & $20 \mathrm{mg}$ & 24-hourly & $8(25.0)$ \\
& $40 \mathrm{mg}$ & 24-hourly & $6(18.8)$ \\
& & 48 -hourly & $2(6.3)$ \\
& $60 \mathrm{mg}$ & 12-hourly & $1(3.1)$ \\
& $80 \mathrm{mg}$ & 24-hourly & $7(21.8)$ \\
& & 12-hourly & $5(15.6)$ \\
& $100 \mathrm{mg}$ & 12-hourly & $1(3.1)$ \\
UFH SC & $5000 \mathrm{IU}$ & 8-hourly & $2(6.3)$ \\
SC = subcutaneous; UFH = unfractionated heparin. &
\end{tabular}

epistaxis, intracranial haemorrhage, including acute subarachnoid haemorrhage and hypertensive thalamic bleed, and/or any form of gastrointestinal bleeding. The most prevalent contraindications were active bleeding during admission and hepatic impairment, which contributed $7.9 \%$ and $3.7 \%$ to the total sample. A total of 17 patients (4.5\%) continued to receive chemoprophylaxis throughout admission despite the presence of documented contraindications to these agents in their medical folders. Contraindications were only detected in 3 patients $(0.8 \%)$ who were prescribed higher doses of enoxaparin (Table 7).

Of the 7 patients with thrombocytopenia, 3 were prescribed enoxaparin $40 \mathrm{mg}$ 24-hourly throughout admission and only one had their regimen altered, being switched to enoxaparin $80 \mathrm{mg}$ 24-hourly. Of those with hepatic impairment, 3 were prescribed enoxaparin $40 \mathrm{mg}$ 24-hourly and 1 received enoxaparin $20 \mathrm{mg}$ 24-hourly. prescribed $(N=32)$ 
Table 7. Contraindications to chemoprophylaxis $(N=380)^{*}$

\begin{tabular}{llll}
\hline & & $\begin{array}{l}\text { Patients who } \\
\text { received chemo- } \\
\text { prophylaxis } \\
\text { throughout } \\
\text { admission, } \boldsymbol{n}(\%)\end{array}$ & $\begin{array}{l}\text { Patients who } \\
\text { were prescribed } \\
\text { high doses of } \\
\text { enoxaparin, }\end{array}$ \\
$\boldsymbol{n}(\%)$
\end{tabular}

Two of the 3 patients who experienced intracranial haemorrhage during admission received enoxaparin $40 \mathrm{mg}$ 24-hourly, while 10 of those who suffered from active bleeding during admission were prescribed enoxaparin $40 \mathrm{mg}$ 24-hourly. Of these 10, 2 had their thromboprophylaxis regimen discontinued entirely and a further 2 were switched to enoxaparin $80 \mathrm{mg}$ and $60 \mathrm{mg} 24$-hourly, respectively.

Significant renal impairment (creatinine clearance $\leq 30 \mathrm{~mL} / \mathrm{min}$ ) was noted in 11 patients (2.9\%). Of these patients, 4 (1.1\%) did not have any form of thromboprophylaxis prescribed, while 6 (1.6\%) were prescribed $40 \mathrm{mg}$ enoxaparin 24-hourly and $1(0.3 \%)$ enoxaparin $60 \mathrm{mg}$ 12-hourly. Two patients $(0.5 \%)$ were switched from enoxaparin $40 \mathrm{mg}$ 24-hourly to $20 \mathrm{mg}$ 24-hourly and 1 (0.3\%) was switched to $40 \mathrm{mg} 48$-hourly.

\section{Discussion}

Appraising VTE risk assessment and prophylaxis practices in medical inpatients is fundamental to understanding the evolution of VTE risk factors and limiting preventable adverse effects and costs associated with inappropriate thromboprophylaxis. Despite the significance of VTE risk assessment and prophylaxis, a paucity of data describing these practices in medical inpatients is evident. The present study therefore aimed to describe these practices in public sector hospitals in the Western Cape. To our knowledge, this study is unique in SA in terms of its evaluation of VTE risk assessment and prophylaxis practices in medical inpatients across multiple public hospitals in the Cape Town metropole. Use of the Caprini RAM to assess VTE risk, correlate risk with recommended thromboprophylaxis, and draw comparisons with what was actually prescribed was also unique in the study setting.

It has been reported that $>75 \%$ of medical inpatients have multiple risk factors for VTE, resulting in an eight times higher risk than that of the general population. ${ }^{[13]}$ This concept is reflected in our findings, where the majority of our patient sample (97.1\%) were determined to be at moderate or higher risk of VTE following objective risk assessment with the Caprini RAM. Similar findings were reported by Shah et al. ${ }^{[14]}$ with $92.7 \%$ of patients found to be at a moderate or high risk of VTE using the Caprini RAM, and by a Cameroonian study, in which $94.6 \%$ of medical inpatients were found to be at risk using the Caprini RAM. ${ }^{[16]}$ The SA TUNE-IN study compared clinical risk assessment using clinician judgement with objective risk assessment using the Caprini RAM. The authors reported a 13.3\% shortfall in the number of patients identified to be at risk for VTE when comparing clinical assessment with the Caprini RAM. ${ }^{[8]}$ Together with our finding that most medical inpatients are at risk for VTE, these results accentuate the need to incorporate structured and validated RAMs into the package of care rendered to medical inpatients.
Obesity (BMI $\geq 30 \mathrm{~kg} / \mathrm{m}^{2}$ ) has been established as an independent risk factor for VTE development. ${ }^{[17]}$ Numerous studies have demonstrated an approximate doubling in VTE risk in obese patients. ${ }^{[18]}$ In our study, the lack of documented weight (21.3\%) and height (0\%) in medical folders was concerning in that BMI determination was excluded. Similar findings were reported in a prospective study at Chris Hani Baragwanath Hospital in Johannesburg, where only 3 out of 352 patients had their weight and height measurements documented. After BMI measurement, the authors found that $16.2 \%$ of their sample were obese. ${ }^{[12]}$ These findings concerning obesity and the lack of BMI measurement are compounded by our finding that $12.4 \%$ of our sample had documented elevated BMI values that were $>25 \mathrm{~kg} / \mathrm{m}^{2}$, yet information required for BMI calculation was lacking. This lack of appreciation for BMI measurement is also evidenced by findings from the TUNE-IN study, where BMI was found to be one of the most overlooked VTE risk factors during risk assessments. ${ }^{[8]}$ Dosing of LMWHs in certain populations is based on BMI, and a paucity of information to calculate it presents various risks to optimal patient health outcomes, including increased bleeding and ineffectual VTE prophylaxis. ${ }^{[9,19]}$ Furthermore, pharmacokinetic studies indicate that a weight-based dosing regimen of enoxaparin could be more effective than a standard fixed-dose regimen in morbidly obese medical inpatients. ${ }^{[20]}$ The use of a standardised VTE RAM could serve to circumvent the lack of BMI measurement, as structured RAMs could prompt clinicians to measure BMI as part of standard risk stratification.

Trends in SA hospital admission data have revealed a shift from infectious diseases as the primary diagnoses to non-communicable diseases. ${ }^{[15,21]}$ In contrast, our study revealed that almost half (49.2\%) of our sample had an infectious disease as the diagnosis. However, our finding may be confounded, as the study period included the period during which the SARS-CoV-2 outbreak and subsequent spread of the virus occurred in SA. ${ }^{[10]}$ This postulation is supported by our finding that $>10 \%$ of the diagnoses identified in our sample were PCR-positive and clinically diagnosed/highly suspected COVID-19 infections.

The recently published findings from the first national $\mathrm{TB}$ prevalence survey in SA demonstrated a high prevalence of the disease, at 737 per 100000 persons. ${ }^{[22]}$ Despite the high prevalence of TB in SA and its strong association with thrombosis, neither the Caprini RAM nor the SA VTE prophylactic and therapeutic guidelines include it as an independent risk factor. ${ }^{[1,9]}$ With regard to TB, the prevalence in our study (17.7\%) was similar to that described by De Vries et al., ${ }^{[23]}$ who reported that $17.2 \%$ of their sample had active TB. Further, Hodkinson and Mahlangu ${ }^{[7]}$ described TB as the predominant VTE risk factor in patients presenting with new-onset DVT in their study. TB as a risk factor for VTE is reported to be poorly understood, despite its known propensity to induce a hypercoagulable state. ${ }^{[24]}$ The added VTE risk conferred by TB is theorised to be linked to prolonged exposure to systemic inflammation, as opposed to acute infections. ${ }^{[25]}$ The causal relationship between TB and thrombosis has also been linked to the hypercoagulable state identified in patients initiating anti-TB treatment. ${ }^{[7]}$ Although the association of TB with VTE development is evidenced by the literature, various RAMs, including the Caprini RAM, neglect to include it as an independent risk factor. This lack of appreciation for TB infection as an independent risk factor is mirrored by the SA VTE prophylactic and therapeutic guidelines, which only allude to anti-TB treatment use as a VTE risk factor. RAMs used in the SA setting should therefore be adapted to include both $\mathrm{TB}$ and anti-TB treatment as VTE risk factors.

HIV infection has a well-established association with thrombosis and consequent VTE development, ${ }^{[7]}$ and the SA VTE prophylactic 
and therapeutic guidelines include HIV infection as a key risk factor, which is noted to confer a high level of VTE risk. ${ }^{\left[{ }^{[0]}\right.}$ Similarly, the Caprini RAM classifies HIV infection under the acquired thrombophilia' section, and infected patients are categorised as being at high VTE risk even in the absence of other risk factors. ${ }^{[1]}$ In our study, acquired thrombophilia as a VTE risk factor comprised HIV infections only, i.e. $27.6 \%$ of our sample were HIV-positive. Similar findings were reported by Du Plooy et al., ${ }^{[21]}$ who found a $29 \%$ prevalence of HIV in their sample. The extensive prevalence of HIV and other infectious diseases in SA should ratify VTE RAM adaptation for use in this setting. A pragmatic approach would be to incorporate a separate HIV subsection into a structured RAM, thus improving its utility. Moreover, a conspicuous HIV subsection could lessen the risk of HIV being overlooked during RAM application.

Numerous studies have demonstrated the strong benefit associated with VTE prophylaxis use in at-risk medical inpatients. ${ }^{[1,26,27]}$ However, thromboprophylaxis remains underutilised in this patient population. ${ }^{[4,8,27]}$ When considering this issue together with the estimation that $75 \%$ of hospitalised patients who die from PE are medical inpatients, the need to improve thromboprophylaxis prescribing is clear. ${ }^{[28]}$ Our findings showed that only $71 \%$ of patients found to be at moderate or higher risk of VTE with the Caprini RAM were prescribed thromboprophylaxis. Similarly, the SA-based TUNE-IN study reported that $73.5 \%$ of medical inpatients in their sample received thromboprophylaxis. ${ }^{[8]} \mathrm{Du}$ Plessis et al. ${ }^{[12]}$ had comparable results, with $73.2 \%$ of those at risk in their sample receiving thromboprophylaxis with an LMWH. In contrast, the ENDORSE study found that just under half (48\%) of medical inpatients in their sample received thromboprophylaxis. ${ }^{[4]}$ Similar findings were reported in a single-centre study in Israel, where 50\% of the at-risk patients in the sample received thromboprophylaxis. ${ }^{[26]}$ However, thromboprophylaxis prescription was markedly better in our study compared with findings from the multicentre DissolVE-2 (Identification of Chinese Hospitalized Patients' Risk Profile for Venous Thromboembolism) study, which found that only $12.9 \%$ of medical inpatients received thromboprophylaxis. ${ }^{[27]}$ Comparable results were described by Nkoke et al., ${ }^{[16]}$ who found that only $18.7 \%$ of high-risk medical inpatients received thromboprophylaxis across two Cameroonian hospitals. Despite our encouraging findings, $29 \%$ of at-risk patients in our sample did not have any form of thromboprophylaxis prescribed. Moreover, almost a quarter (24\%) of at-risk patients did not receive thromboprophylaxis despite their lack of contraindications to chemoprophylaxis. On the basis of these findings, it may be construed that thromboprophylaxis prescribing in medical inpatients is expanding. However, a large number of at-risk patients are still overlooked, which may be attributed to a lack of objective RAM use in our setting.

Our finding that enoxaparin $40 \mathrm{mg}$ 24-hourly was prescribed in $89 \%$ of our sample was anticipated, as this is the standard thromboprophylaxis regimen in most public healthcare sector hospitals in the Western Cape. ${ }^{[29,30]}$ Dosing anomalies (doses $>40 \mathrm{mg}$ and frequencies differing from 24-hourly) were detected in $15 \%$ of patients who were prescribed thromboprophylaxis. Du Plessis et al. ${ }^{[12]}$ reported similar findings, with $17.5 \%$ of their sample noted to have received the incorrect dose of an LMWH. These findings may be indicative of a new trend in VTE prophylaxis prescribing, where rates of thromboprophylaxis prescribing in medical inpatients are increasing, but inappropriate dosing is increasing as a consequence. This trend may be further complicated by the inappropriate prescribing of chemoprophylaxis in patients with contraindications, as evidenced by the $4.5 \%$ of patients in our sample who received chemoprophylaxis throughout admission. Comparable findings were reported by Rocher et al., ${ }^{[2]}$ who found that $5.6 \%$ of patients in their sample were prescribed some form of chemoprophylaxis despite clear contraindications.

Mechanical thromboprophylaxis is a fundamental therapy for preventing VTE in patients with contraindications to anticoagulants, such as active bleeding. ${ }^{[4]}$ The Caprini RAM recommends mechanical prophylaxis as an alternative to chemoprophylaxis in patients at moderate VTE risk and as adjuvant therapy in those considered to be at high and highest risks. ${ }^{[31]}$ No prescription for any form of mechanical thromboprophylaxis was identified in our study. Our finding was concordant with findings from other African studies, where a complete lack of mechanical thromboprophylaxis prescriptions was identified. ${ }^{[5,16]}$ This paucity of mechanical thromboprophylaxis prescribing is worrying, owing to the number of at-risk patients in our sample who had contraindications to chemoprophylaxis and would have benefited from this form of prophylaxis (13.4\%). This lack of mechanical prophylaxis prescribing is further compounded by our finding that $39.2 \%$ of patients with documented contraindications to chemoprophylaxis nevertheless had anticoagulants prescribed. However, the possible lack of available mechanical thromboprophylaxis equipment for medical inpatient use may be a factor contributing to these findings. The use of IPC in resource-limited settings such as public healthcare sector hospitals is challenging, as these devices require maintenance to ensure optimal functionality. However, GCSs may offer a more feasible approach for use in medical inpatients and require further consideration. The need to improve access to and awareness of mechanical thromboprophylaxis use in medical inpatients is evident from our findings.

\section{Study limitations}

The retrospective nature of this study is a key limitation, since the quality of data obtained depends on the accuracy and quality of information documented in medical folders. The hospitals selected for this study were all located in the Cape Town metropole, and VTE risk assessment and prophylaxis practices may differ in rural facilities with limited access to specialist clinician consultations.

\section{Conclusions}

An improvement in the rate of thromboprophylaxis prescribing in medical inpatients is supported by our findings. However, a substantial portion of at-risk patients (29\%) are still overlooked in practice, validating the need for extensive appropriation of structured RAMs in the SA public healthcare sector. Furthermore, our study uncovered a consequence of this improvement, where inappropriate dosing of anticoagulants is expanding. The issue of inappropriate dosing of anticoagulants is further complicated by the lack of mechanical prophylaxis prescribing as evidenced by our findings. The use of mechanical prophylaxis should be prioritised to bolster awareness around the benefits of use in patients with contraindications to anticoagulants. TB should be recognised as an independent risk factor for VTE, owing to its propensity to induce thrombosis and its extensive prevalence in SA. Together with HIV, TB and anti-TB treatment should be incorporated into structured RAMs for use in the SA setting. Demarcated subsections for HIV and TB should be incorporated into RAMs to improve utility and convenience of use. The Caprini RAM offers a validated, simplistic and effective approach to VTE risk assessment in medical inpatients. The Caprini RAM may be refined and adapted for specific use in public healthcare sector hospitals in the Cape Town metropole. Novel research should explore reasons underpinning the lack of VTE prophylaxis prescribing and inappropriate prescribing of anticoagulants in medical inpatients. 
Declaration. The research for this study was done in partial fulfilment of the requirements for AW's M Clin Pharm degree at the University of the Western Cape.

Acknowledgements. We acknowledge and thank Yasmina Johnson for her help in accessing healthcare facilities for data collection. We also thank the pharmacy managers at the participating facilities for their logistical and administrative support.

Author contributions. AW conceptualised and designed the study, performed data collection and analysis and drafted the manuscript. RC and JM supervised the study, aided in the design of the study and the data collection tool, and were major contributors to the final manuscript.

Funding. None.

\section{Conflicts of interest. None.}

1. Cronin M, Dengler N, Krauss ES, et al. Completion of the updated Caprini risk assessment model (2013 version). Clin Appl Thromb Hemost 2019;25:1076029619838052. https://doi. org/10.1177/1076029619838052

2. Rocher WD, Page T, Rocher M, et al. Venous thromboembolism risk and prophylaxis prescription in surgical patients at a tertiary hospital in Eastern Cape Province, South Africa. S Afr Med J 2019;109(3):178-181. https://doi.org/10.7196/SAMJ.2019.v109i3.13510

3. Van der Merwe M, Julyan M, Du Plessis JM. Is guideline-driven prophylaxis for venous thromboembolism common practice in the South African private hospital setting? S Afr Fam Pract 2020;62(1):el-e9. https://doi.org/10.4102/safp.v62i1.5022

4. Cohen AT, Tapson VF, Bergmann JF, et al. Venous thromboembolism risk and prophylaxis in the acute hospital care setting (ENDORSE study): A multinational cross-sectional study. Lancet 2008;371(9610):387-394. https://doi.org/10.1016/S0140-6736(08)60202-0

5. Kingue S, Bakilo L, Ze Minkande J, et al. Epidemiological African day for evaluation of patients at risk of venous thrombosis in acute hospital care settings. Cardiovasc J Afr 2014;25(4):159-164. https://doi. org/10.5830/CVJA-2014-025

6. Naidoo P, Mothilal R, Snyman LC. Assessment and management of venous thrombo-embolism risk during pregnancy and the puerperium (SAVE): The South African cohort. S Afr Med J 2019;109(3):186192. https://doi.org/10.7196/SAMJ.2019.v109i3.13487

7. Hodkinson KE, Mahlangu JN. Deep-vein thrombosis in the era of high HIV and tuberculosis prevalence: A prospective review of its diagnosis and treatment in a quaternary centre. S Afr Med J 2017;107(10):859-863. https://doi.org/10.7196/SAMJ.2017.v107i10.12443

8. Wessels P, Riback WJ. DVT prophylaxis in relation to patient risk profiling - TUNE-IN study. S Afr Med J 2012;102(2):85-89. https://doi.org/10.7196/SAMJ.4859

9. Jacobson BF, Louw S, Büller H, et al. Venous thromboembolism: Prophylactic and therapeutic practice guideline. S Afr Med J 2013;103(4 Pt 2):261-267. https://doi.org/10.7196/SAMJ.6706

10. Loveday M, Cox H, Evans D, et al. Opportunities from a new disease for an old threat: Extending COVID-19 efforts to address tuberculosis in South Africa. S Afr Med J 2020;110(12):1160-1167. https://doi.org/10.7196/SAMJ.2020.v110i12.15126

11. Legenza L, Barnett S, Rose W, et al. Epidemiology and outcomes of Clostridium difficile infection among hospitalised patients: Results of a multicentre retrospective study in South Africa. BMJ Glob Health 2018;3(4):e000889. https://doi.org/10.1136/bmjgh-2018-000889

12. Du Plessis JA, van Blydenstein SA, Wong M. Evaluation of the use of low-molecular-weight heparin for venous thromboembolism prophylaxis in medical patients. S Afr Med J 2020;110(3):235-242. https:// doi.org/10.7196/SAMJ.2020.v110i3.14279
13. Skeik N, Westergard E. Recommendations for VTE prophylaxis in medically ill patients. Ann Vasc Dis 2020:13(1):38-44. https://doi.org/10.3400/avd.ra. 19-00115

14. Shah SS, Abdi A, Özcem B, et al. The rational use of thromboprophylaxis therapy in hospitalised patients and the perspectives of health care providers in Northern Cyprus. PLoS ONE 2020;15(7):e0235495.
and https://doi.org/10.1371/journal.pone.0235495

15. Roche S, de Vries E. Multimorbidity in a large district hospital: A descriptive cross-sectional study. S Afr Med J 2017;107(12):1110-1115. https://doi.org/10.7196/SAMJ.2017.v107i12.12397

16. Nkoke C, Tchinde Ngueping MJ, Atemkeng F, et al. Incidence of venous thromboembolism, risk factors and prophylaxis in hospitalised patients in the south west region of Cameroon. Vasc Health Risk Manag 2020;16:317-324. https://doi.org/10.2147/VHRM.S205935

17. Kaze AD, Bigna JJ, Nansseu JR, et al. Body size measures and risk of venous thromboembolism: Protocol for a systematic review and meta-analysis. BMJ Open 2018;8(3):e018958. https://doi. org/10.1136/bmjopen-2017-018958

18. Klovaite J, Benn M, Nordestgaard BG. Obesity as a causal risk factor for deep venous thrombosis A Mendelian randomization study. J Intern Med 2015;277(5):573-584. https://doi.org/10.1111/ joim. 12299

19. Minze MG, Kwee Y-Y, Hall RG II. Low-molecular-weight heparin prophylaxis dosing: Is weight an issue? J Pharm Technol 2016;32(2):75-80. https://doi.org/10.1177/8755122515617200

20. Rondina MT, Wheeler M, Rodgers GM, et al. Weight-based dosing of enoxaparin for VTE prophylaxis in morbidly obese, medically-ill patients. Thromb Res 2010;125(3):220-223. https://doi.org/10.1016/j. thromres.2009.02.003

21. Du Plooy N, Day C, Manning K, et al. Prevalence and outcome of delirium among acute general medical inpatients in Cape Town, South Africa. S Afr Med J 2020;110(6):519-524. https://doi. org/10.7196/SAMJ.2020.v110i6.14363

22. Pillay Y, Mvusi L, Mametja LD, Dlamini S. What did we learn from South Africass first-ever tuberculosis prevalence survey? S Afr Med J 2021;111(5):402-404. https://doi.org/10.7196/SAMJ.2021. v111i5.15662

23. De Vries E, Raubenheimer P, Kies B, et al. Acute hospitalisation needs of adults admitted to public facilities in the Cape Town Metro district. S Afr Med J 2011;101(10):760-764. https://doi.org/10.7196/ SAMJ.4586

24. Awana PA, Danwang C, Tochie JN, et al. Epidemiology of venous thromboembolism in people with active tuberculosis: A systematic review and meta-analysis protocol. BMJ Open 2019;9(11):e031402. https://doi.org/10.1136/bmjopen-2019-031402

25. Borjas-Howard JF, Bierman WFW, Meijer K, et al. Venous thrombotic events in patients admitted to a tuberculosis centre. Q J Med 2017;110(4):215-218. https://doi.org/10.1093/qimed/hcw152 26. Koren $\mathrm{O}$, Nasser A, Elias $\mathrm{M}$, et al. Low venous thromboembolism incidence in high risk medical
patients in an Israeli hospital: Can risk assessment be extrapolated to different populations? PLoS ONE 2020;15(7):e0235683. https://doi.org/10.1371/journal.pone.0235683

27. Zhai Z, Kan Q, Li W, et al. VTE risk profiles and prophylaxis in medical and surgical inpatients: The Identification of Chinese Hospitalized Patients' Risk Profile for Venous Thromboembolism (DissolVE-2) - a cross-sectional study. Chest 2019;155(1):114-122. https://doi.org/10.1016/j. chest.2018.09.020

28. Cohen AT, Nandini B, Wills JO, et al. VTE prophylaxis for the medical patient: Where do we stand? a focus on cancer patients. Thromb Res 2010;125(Suppl 2):S21-S29. https://doi.org/10.1016/s00493848(10)70008-6

29. National Department of Health, South Africa. Standard Treatment Guidelines and Essential Medicines List: Hospital Level (Adults). 5th ed. 2019. https://www.knowledgehub.org.za/elibrary/hospital-level adults-standard-treatment-guidelines-and-essential-medicines-list-2nd (accessed 4 October 2021).

30. Jacobson BF, Louw S, Riback W. The Use of VTE prophylaxis in relatioN to patiEnt risk profiling (TUNE-IN) wave 2 study. S Afr Med J 2014;104(12):880-884. https://doi.org/10.7196/SAMJ.8456

31. Caprini JA. Thrombosis risk assessment as a guide to quality patient care. Dis Mon 2005;51(2-3):70-78 https://doi.org/10.1016/j.disamonth.2005.02.003

Accepted 3 November 2021 